ELENA MAZZOLENI

ORCID: 0000-0003-2499-8015

Università degli Studi di Bergamo

elena.mazzoleni@unibg.it

\title{
LES FOURBERIES DE LA FOIRE : UNE RÉVOLUTION SOCIOPOLITIQUE ET THÉÂTRALE
}

La rivalité entre les théâtres parisiens officiels et les tréteaux populaires, tels que les loges de la Foire et les salles de boulevard, contribue à développer, entre les $\mathrm{XVI}^{\mathrm{e}}$ et $\mathrm{XIX}^{\mathrm{e}}$ siècles, une dramaturgie expérimentale caractérisée par la prééminence du geste de l'acteur, l'hybridation et la flexibilité scéniques. Pour contourner les interdictions imposées par la Comédie-Française et l'Académie Royale de Musique, les imprésarios forains élaborent des stratégies dramaturgiques pleines d'essor construites sur des pièces à unités simples et aptes à l'intersection des genres. Ces spectacles sont des témoignages, d'abord, de la contamination entre la tradition de la Commedia dell'Arte et le répertoire populaire français; ensuite, de la prise de conscience des enjeux créatifs de la dramaturgie non officielle, et enfin, de la consécration du geste de l'acteur. Le théâtre populaire s'avère en mesure de se développer en un système artistique stable, malgré un contexte marqué par la marginalité et l'incertitude; et d'inspirer certaines théories du $\mathrm{XX}^{\mathrm{e}}$ siècle visant à réformer le théâtre à travers le corps de l'acteur et l'implication des spectateurs, comme celles d'Edward Gordon Craig et de Jacques Copeau.

Dans le sillon de certaines études consacrées au théâtre populaire français, à savoir celles de Pauline Beaucé, Renzo Guardenti, Roxane Martin, Françoise Rubellin et David Trott ${ }^{1}$, nous envisageons d'étudier des témoignages documentaires

${ }^{1}$ P. Beaucé, Parodies d'opéra au siècle des Lumières : évolution d'un genre comique, Presses Universitaires de Rennes, coll. "Le Spectaculaire Théâtre », Rennes 2013 ; eadem, "Les Jeux de mots dans le répertoire des théâtres de la Foire à Paris au XVIII ${ }^{\mathrm{e}}$ siècle : de la publicité à la satire ", 
d'archive non seulement pour mettre en lumière la spécificité de ces répertoires non officiels, mais aussi pour tracer une carte des migrations spectaculaires qui fasse ressortir les tréteaux de la Foire comme des lieux d'affrontement sociopolitique, et en même temps, des systèmes de propagation des réformes esthétiques. Ce parcours portera, enfin, sur l'évidence qu'il n'existe aucune opposition nette entre tradition et innovation, et qu'au contraire, le conflit entre la censure et la transgression du canon, propre au théâtre populaire, est nécessaire à tout geste artistique.

La période de 1680 à 1720 est très importante pour le théâtre français : la fondation, la consolidation des scènes officielles (telles que la Comédie-Française, l'Opéra, la Comédie-Italienne) et l'attribution consécutive des privilèges en fonction des genres à représenter sont désormais accomplies ${ }^{2}$. Un circuit fermé se crée, qui, d'un côté, valide la centralisation du pouvoir, et de l'autre, empêche la formation et le développement d'organisations théâtrales alternatives. Des tensions acharnées ne cessent par la suite d'investir le théâtre lié au pouvoir et les tréteaux populaires, qui pourtant attirent un public socialement mixte. En effet, les approches sociologiques plus récentes sollicitent une réflexion sur la valeur attribuée à l'adjectif « populaire », qu'il est opportun d'employer dans son acception la plus nuancée ${ }^{3}$.

Le 14 mai 1697, une ordonnance émise par Louis XIV prend effet. Elle met fin à l'activité artistique de la compagnie italienne, qui depuis longtemps exerçait un rôle majeur au sein de la vie théâtrale grâce à une dramaturgie à fort impact

[dans :] Enjeux du jeu de mots. Perspectives linguistiques et littéraires, E. Winter-Froemel et A. Zirker (dir.), De Gruyter, Berlin-Boston 2015, pp. 65-80 ; et avec F. Rubellin, Parodier l'opéra : pratiques, formes et enjeux, Éditions Espaces 34, Les Matelles 2015 ; R. Guardenti, « Drammaturgia e guerriglie teatrali dall'Ancien Théâtre Italien alla Foire ", [dans :] Il teatro allo specchio: il metateatro tra melodramma e prosa, Turchini Edizioni, Napoli 2008, pp. 87-102 ; idem, Le fiere del teatro. Percorsi del teatro forain del primo Settecento. Con una scelta di commedie rappresentate alle Foires Saint-Germain e Saint-Laurent, Bulzoni, Roma 1995 ; idem, « Il teatro e il suo contesto: le fiere di Saint-Germain e Saint-Laurent », Biblioteca teatrale XXX-XXXII, 1993, pp. 93-121 ; idem, «Per le vie della provincia: i comici italiani e La Vengeance de Colombine di Nicolas Barbier», Biblioteca teatrale XXXV, 1992, pp. 1-36, et Gli italiani a Parigi. La Comédie Italienne (1660 1697). Storia, pratica scenica, iconografia, Bulzoni, Roma 1990 ; R. Martin, " Le Théâtre de la foire : des tréteaux aux Boulevards ", Studies on Voltaire and the Eighteenth Century X, Oxford 2002 ; Ris, masques et tréteaux. Aspects du théâtre du XVIII siècle. Mélanges en hommage à David A. Trott, études réunies et éditées par M.L. Girou-Swiderski, S. Massé et F. Rubellin, Presses de l'Université de Laval, Québec 2008 ; Théâtre de la Foire. Anthologie de pièces inédites 1712-1736, F. Rubellin (dir.), Éditions Espaces 34, Montpellier 2005, et F. Rubellin, «L'écriture des lazzi dans le théâtre forain au XVIII ${ }^{\mathrm{e}}$ siècle », Revue d'histoire du théâtre I-II, 2010, pp. 173-183 ; D. Trott, Théâtre du XVIII siècle : jeux, écritures, regards. Essai sur les spectacles en France de 1700 à 1790, Éditions sociales, Montpellier 1978.

2 Ces années qui correspondent à la fin du règne de Louis XIV sont marquées par un fanatisme religieux sous l'influence de $\mathrm{M}^{\mathrm{me}}$ de Maintenon. Cela explique certaines décisions prises au détriment des acteurs italiens.

3 À ce propos, les références sont nombreuses ; nous renvoyons, entre autres, aux études de Pierre Bourdin et de David Trott. 
visuel. La première de la comédie La Fausse Prude, avec ses prétendues allusions à $\mathrm{M}^{\mathrm{me}}$ de Maintenon, fournit le prétexte pour éloigner de la scène les acteurs italiens. Toutefois, leur expulsion n'entraîne pas la suppression de leur répertoire ; au contraire, en plus de contribuer au développement de la farce française, celui-ci « migre » vers les loges des foires. Les imprésarios de Saint-Germain et de Saint-Laurent, et plus tard ceux des théâtres de boulevard se considèrent, en effet, les principaux héritiers de la dramaturgie italienne qui est, en quelque sorte, semblable à la leur, car elle repose sur les effets de scène et sur l'interprétation de l'acteur ${ }^{4}$.

Bien qu'elles aient été fondées pour permettre l'échange de marchandises, les deux principales foires parisiennes présentent d'emblée une vaste offre théâtrale. La Plainte du Carnaval et de la Foire de Saint-Germain en vers burlesques (1649), une mazarinade anonyme défendant les fêtes de carnaval, nous donne un aperçu sur la foire d'hiver de Saint-Germain :

[...] l'on voyoit tous les hyvers

Les raretez que l'univers

Produit dedans chaque contrée

Des sauteurs, des faiseurs de tours,

Des hommes qui monstrent des ours,

Des singes, des Marionnettes,

Et mille conteurs de sonnettes,

Et lors que l'on estoit dedans

L'on y voyoit autant de gens

Que de sortes de marchandises,

De meubles, \& de friandises :

C'est là qu'on voyoit des Tableaux,

D'hommes, de bestes, \& d'oiseaux 5 .

${ }^{4}$ G. Attinger, L'Esprit de la commedia dell'arte dans le théâtre français, Slatkine, Genève 1993 [1950] ; H. Lagrave, « La Pantomime à la Foire, au Théâtre Italien et aux Boulevards (17001789) ", Romantische Zeitschrift für Literaturgeschichte III-IV, 1979, pp. 408-450 ; R. Lelièvre, Le Théâtre dramatique italien en France (1855-1940), Colin, Paris 1959; La Commedia dell'Arte, le théâtre forain et les spectacles de plein air en Europe (XVI-XVIII siècles), I. Mamczarz (dir.), Klincksieck, Paris 1998 ; C. Parfaict et F. Parfaict, Histoire de l'Ancien Théatre Italien depuis son origine en France jusqu'à sa suppression en l'année 1697. Suivie des extraits ou canevas des meilleures pièces italiennes qui n'ont jamais été imprimées, Rozet, Paris 1767, et iidem, Histoire du Théâtre Français depuis son origine jusqu'à présent, Le Mercier-Saillant, Paris 1745-1749.

5 Anonyme, "Plainte du Carnaval et de la Foire de Saint-Germain en vers burlesques », [dans :] Nouveau journal contenant tout ce qui s'est fait \& passé aux Assemblées des compagnies Souveraines du Parlement de Paris, ès années 1648-1649 jusqu'à présent, Colombel, Paris 1649, p. 7. Cet exemplaire est conservé à la Bibliothèque de l'Arsenal de Paris (H 7164). Les mazarinades sont des textes poétiques de nature et style très différents : ce sont des pièces en vers burlesques ou des pamphlets en prose publiés pendant la Fronde et adressés contre le cardinal Mazarin. Parmi leurs auteurs figurent le cardinal de Retz, Saint-Amant, Loret, Sarrasin, Guy Patin, Laffemas, Patru et Cyrano de Bergerac. Dans ce poème, le cardinal Mazarin est décrit comme celui qui « fit icy venir de si loin / À force d'argent, \& de soin / De ridicules personnages / Avec de lascives images. / Quoy ce Zany, ce Pantalon / Ce Phorphante, ce Violon, / Ce jongleur qu'on déguize en Pretre [...] 
Acrobates, marionnettistes et autres montreurs de bizarreries (objets de cire, animaux dressés, géants, nains, automates et momies) occupent donc les loges de la Foire. Toutefois, une véritable dramaturgie ne se développera pas avant 1595 , lorsque seront enfin représentées certaines parades en dialogues. C'est seulement vers 1618 que les premiers acteurs ambulants recevront l'autorisation de la Confrérie de la Passion de présenter des numéros acrobatiques à caractère dramaturgique minimal. Tout au long du XVII ${ }^{\mathrm{e}}$ siècle, les compagnies actives dans les deux foires sont seulement au nombre de trois : celle des frères Allard, celle de l'acrobate Maurice van der Beck et celle du montreur de marionnettes Alexandre Bertrand.

Vers la fin du XVII ${ }^{\mathrm{e}}$ siècle, le théâtre de la Foire, du fait de son succès croissant, commence à être perçu comme une menace, surtout à cause de ses entractes chantés, qui défient le monopole de l'Opéra ${ }^{6}$. La Comédie-Française, bénéficiaire du privilège exclusif du « droit de parole », entame une longue et dure opposition contre les activités foraines, recourant systématiquement au Parlement pour rendre effectifs des interdits qui empêchent la représentation de farces et comédies. Le régime des privilèges, soutenu sur le plan théorique par la division classique des genres instaurée par l'Art pratique du théâtre (1657) de l'abbé d'Aubignac et par l'Art poétique (1674) de Nicolas Boileau, permet l'exclusivité des représentations ; ce qui garantit la continuité du processus de centralisation de la culture, mais qui est aussi une entrave aux spectacles populaires. Toutefois, le théâtre de la Foire réussira à supporter ce conflit avec les théâtres officiels grâce, en premier lieu, à une dramaturgie basée sur des structures flexibles ; à la possibilité de puiser dans la tradition italienne désormais codifiée ; à l'introduction d'éléments musicaux; et enfin, à l'utilisation de procédés scéniques originaux et gagnants. Courtes scènes de parodie monologuées ${ }^{7}$, comédies à la muette, pantomimes, vaudevilles sont parmi les solutions les plus ingénieuses adoptées pour

Ce detestable Cardinal / Outre le festin, \& le bal / Prive Paris de ses délices, / Luy qui n'aime rien que les vices. / [...] Le Careme, \& le Carnaval / Feront la guerre au Cardinal» (ibidem, pp. 7-8). L'auteur (peut-être P. Scarron) fait allusion au cardinal de manière explicite car il évoque sa vanité, ses dépenses exagérées pendant la guerre de Trente Ans, ses mauvaises mœurs, son goût pour les œuvres d'art, comme celles du Corrège et du Véronèse, et enfin son penchant pour le théâtre italien. On sait que le chorégraphe Giovan Battista Balbi et le scénographe Giacomo Torelli ont été appelés en France par Mazarin même.

${ }^{6}$ Sur l'histoire du théâtre de la Foire et son répertoire, voir M. Albert, Les Théâtres de la Foire (1660-1789), Hachette, Paris 1900 ; É. Campardon, Les Spectacles de la Foire. Théâtres, acteurs, sauteurs et danseurs de corde, monstres, géants, nains, animaux curieux ou savants, marionnettes, automates, figures de cire et jeux mécaniques des Foires Saint-Germain et Saint-Laurent, des Boulevards et du Palais-Royal depuis 1595 jusqu'à 1791. Documents inédits recueillis aux Archives nationales, 2 vol., Slatkine, Genève 1970 ; et A. Heulhard, La Foire de Saint Laurent. Son histoire et ses spectacles, Slatkine, Genève 1971 [1877].

7 Cf. E. Mazzoleni, « Momus at the Fairs and the Early Boulevard Theatres: Parody in French Plays from the Ancien Régime to the Restoration Period », Elephant \& Castle XV, 2016, pp. 5-28. 
contourner les restrictions imposées par les institutions théâtrales, qui possèdent le monopole des représentations.

À partir de 1703, les interdits deviennent de plus en plus sévères : les forains peuvent jouer seulement des scènes distinctes (de brèves séquences dialoguées sans lien entre elles), de façon à les exclure de l'idéal classique des trois unités. Au cours des années suivantes, les sentences déjà émises sont confirmées : la représentation des comédies dialoguées est interdite. Les artistes forains répondent par des monologues qui se développent de deux façons : soit les acteurs sont sur scène, mais seulement l'un d'entre eux profère des mots tandis que les autres s'expriment à travers gestes et pantomimes, soit les comédiens parlent à voix haute depuis les coulisses. Ces pièces, bien qu'entortillées, réussissent à séduire le public. Face au succès des salles populaires, les acteurs officiels décident d'avoir recours à des interdits encore plus radicaux, comme l'imposition du silence. Les forains réagissent de façon hautement significative du point de vue de l'expérimentation théâtrale. Tel est le cas de l'imprésario théâtrale Jeanne Godefroy (la femme de Maurice van der Beck). Associée depuis 1699 avec les frères Allard dans la gestion du Jeu de paume du duc d'Orléans, elle négocie en 1708 avec l'Opéra l'autorisation de mettre en scène de brefs interludes avec des airs musicaux. Cette échappatoire mènera à la fondation de l'Opéra-Comique, et de manière générale, à l'utilisation de structures dramatiques mixtes composées de couplets chantés et de parties en prose, propres à satisfaire le goût du public, qui participe ainsi plus activement au développement du spectacle.

Durant la période de 1703 à 1710, période la plus dure des interdits, qui entraînent souvent la fermeture de nombreuses salles, la scène foraine met au point une échappatoire dramaturgique remarquable : la comédie à écriteaux. La gestuelle pantomimique, la richesse chorégraphique et la complicité du public sont à la base de ces représentations. Le texte des acteurs est affiché en grands caractères sur des panneaux défilant progressivement depuis le plafond de la salle, et les répliques sont remplacées par des airs en vers. L'orchestre exécute l'air et certains acteurs présents dans la salle suggèrent les paroles au public ; celui-ci participe au déroulement du spectacle. Les didascalies et les avis aux lecteurs permettent d'enrichir et d'accompagner la performance, donnant ainsi au geste toute sa valeur expressive ${ }^{8}$.

La contamination de la dramaturgie italienne est une autre particularité du théâtre de la Foire qui, entre 1707 et 1721, met en scène pas moins d'une vingtaine de comédies d'inspiration italienne, essentiellement tirées du recueil d'Evaristo Gherardi, Le Théâtre Italien ou Le recueil général de toutes les scenes françoises qui ont eté joüées sur le Théâtre-Italien de l'Hostel de Bourgogne (1694). La célèbre comédie Arlequin, roi de Serendib (1713) composée par Lesage pour la

${ }^{8}$ Pour une étude sur les pièces à écriteaux, voir en particulier P. Martinuzzi, Les Pièces par écriteaux nel teatro della Foire (1710-1715). Modi di una teatralità, Cafoscarina, Venezia 2007. 
compagnie de la Dame Baron confirme le lien entre la tradition italienne et le théâtre forain. En particulier, la rencontre entre Arlequin et quelques voleurs, donnant lieu d'abord à un cambriolage, puis à un banquet et enfin à un mauvais tour au détriment du personnage masqué, représente bien le recours à une interprétation exclusivement fondée sur le geste de l'acteur et sur les lazzi (les fourberies) de la Commedia dell'Arte :

Les Voleurs prennent le tonneau, le défoncent, y mettent Arlequin, et s'en vont, après avoir remis les fonds. Arlequin se voyant sans espérance de salut, pleure, crie, en roulant son tonneau. Il vient un Loup affamé qui cherche de la pâture. Il va flairer le tonneau; et comme il y sent de la chair fraîche, il fait tous ses efforts pour en briser les douves. Pendant qu'il s'y prend de toutes les manières, Arlequin passe la main par le trou de la bonde, attrape la queue du Loup, qui se voyant saisi, a peur et veut prendre la fuite ; mais en tirant le tonneau, sa queue demeure entre les mains d'Arlequin, et dans le moment le tonneau se partage en deux. Le Loup se sauve d'un côté, et Arlequin de l'autre'.

Ce n'est pas un hasard si, dans ces années-là, de nombreux auteurs français actifs dans les théâtres officiels prêtent volontiers leur plume aux acteurs italiens et aux imprésarios de la Foire. Jean-François Regnard et Charles Dufresny publient les recueils Scènes françoises de la Comédie Italienne, intitulée "La foire S. Germain » (1696), Le Théâtre Italien ou Recueil de toutes les comédies et scènes françaises qui ont été jouées sur le théâtre Italien par la troupe des comédiens du Roy de l'Hôtel de Bourgogne à Paris (1695), ainsi que La Foire de Saint Germain, comédie françoise et italienne, en trois actes et en prose, mêlée de vers (26 décembre 1695$)^{10}$.

La comédie est située à la foire de Saint-Germain, où le docteur est à la recherche de sa bien-aimée Angélique, qui s'attarde au milieu des loges de la foire avec Colombine, Arlequin et Pierrot. Ici, la jeune fille rencontre Octave dont elle tombe amoureuse. Selon l'intrigue traditionnelle de la Commedia dell'Arte, les serviteurs (Arlequin et Pierrot) favorisent le mariage des amoureux au détriment des projets du docteur. Des scènes comiques dépeignent l'ambiance de la foire comme un univers corrompu, où l'on assiste à des spectacles mixtes mêlant vaudevilles, passages de nature méta-théâtrale et parodies (l'une de l'opéra Acis et Galatée de Lully, et l'autre de la tragédie Lucrèce de Reyer). Dans le troisième et dernier acte, Pierrot et Arlequin soutiennent le dessein d'Octave d'épouser Angélique. Ensemble ils réfléchissent à une solution pour contourner le docteur, et il s'ensuit un échange dans le style de la Commedia dell'Arte. Pierrot, le zanni impertinent, propose de rendre le docteur muet : "Voici comment j'argumente :

9 A.R. Lesage, «Arlequin roi de Serendib», [dans :] Théâtre de la foire, Ganeau, Paris 17211737, p. 9 (acte I, scène première).

${ }^{10}$ Nous faisons référence à une édition postérieure, datant du dix-huitième siècle, conservée à la Bibliothèque de l'Arsenal de Paris (EG 10727). Dans l'avertissement, l'éditeur informe qu'il existe une version réduite de la pièce imprimée à Grenoble en 1696 et signée par Florent Carton Dancourt. L'exemplaire de 1696 est conservé à la Bibliothèque de l'Arsenal de Paris (EGC 23 273). 
Qui est muet, ne dit mot ; qui ne dit mot, consent. Ergó, en rendant le docteur muet, nous aurons son consentement $\rangle^{11}$.

À partir de la seconde moitié du XVIII ${ }^{\mathrm{e}}$ siècle, le théâtre de la Foire occupe les salles de la périphérie parisienne sans pourtant renoncer à des formes de représentations expérimentales basées sur des schémas de la Commedia dell'Arte mêlant thèmes méta-théâtraux, épisodes satiriques et parodies concernant l'actualité, aussi bien que des intrigues situées dans un univers mythologique et exotique. En 1759, l'imprésario de théâtre Jean-Baptiste Nicolet, abandonne les loges foraines pour une activité stable sur le boulevard du Temple. Son offre artistique, dès le départ très hétérogène, inclut des spectacles de funambules, pantomimes et marionnettes, puis, à partir de 1764, se spécialise dans l'opéra-comique et les arlequinades de style italien. Suivant le modèle de ce théâtre, une migration dramaturgique commence alors depuis les foires vers les quartiers périphériques. En quelques années et dans un espace urbain limité, on voit fleurir de nombreuses salles, toutes caractérisées par une offre alternative par rapport à celle subventionnée par le pouvoir. Face au succès croissant de ces salles (ou plus probablement en vertu de leur fortune), le conflit avec le système théâtral institutionnel, plutôt que de s'atténuer, s'accentue.

Pendant la décennie révolutionnaire, cette rivalité ne cessera d' « enflammer » les scènes. Malgré les transformations qui ont vu les spectacles de la Foire passer par tant de formes, quelque chose a perduré du point de vue du répertoire et du style. Parmi les persistances, il y a plusieurs salles (par exemple le théâtre d'Audinot, qui est détruit par un incendie en mai 1798), des artistes dont les origines remontent aux derniers moments de l'entreprise foraine, et des fragments d'un répertoire aux racines parfois traçables mais, conformément à l'essence des représentations théâtrales, toujours en évolution. Par exemple, les spectacles du Théâtre du Vaudeville s'orientent vers ceux du théâtre forain, d'abord par l'adaptation de pièces telles que Nicaise de Vadé (le 18 janvier 1792), La Chercheuse d'esprit de Favart (le 24 août 1793) et Le Nouveau magasin des modernes, adaptation du Magasin des modernes de Pannard (le 8 décembre 1798) ${ }^{12}$. Une continuité qui implique donc une évolution perpétuelle. Entre 1789 et 1800, le théâtre de la Foire survit en tant que forme de spectacle qui traverse les genres, les répertoires, les styles et les changements socio-culturels que connait le public ${ }^{13}$.

11 F.R. Regnard et C. Dufresny, La Foire de Saint Germain, 1696, p. 235.

12 Cf. D. Trott, op. cit.

$13 C f$. les études incontournables de $\mathrm{P}$. Bourdin à propos du théâtre révolutionnaire. Nous renvoyons entre autres à " Le théâtre de la Foire. Des tréteaux aux boulevards », Annales historiques de la Révolution française 337, 2004, pp. 201-202; Les arts de la scène et la Révolution française, Presses universitaires Blaise Pascal, Clermont-Ferrand 2004 ; «Au théâtre sous la Terreur », [dans :] Visages de la Terreur, M. Biard et H. Leuwers (dir.), A. Colin, Paris 2004 ; avec F. Le Borgne, Costumes, décors et accessoires dans le théâtre de la Révolution et de l'Empire, Presses universitaires Blaise Pascal, Clermont-Ferrand 2010 ; avec M. Biard, La France en révolution (1787-1799), 
Bien que la Comédie-Française et l'Opéra persistent dans la défense de la séparation des genres, dans la revendication de leur privilège de représentation et dans l'élimination de la dimension gestuelle, tout au long du XIX ${ }^{\mathrm{e}}$ siècle, une dramaturgie hybride ne cesse de se développer en parallèle, mêlant comédies, pantomimes, acrobaties, vaudevilles chantés et dansés.

Un des nombreux exemples est donné par Les Écriteaux, ou René Le Sage à la Foire de Saint-Germain, fruit de la collaboration entre Barré, Desfontaines et Radet. Il s'agit d'un spectacle en deux actes construit sur l'entrecroisement de dialogues et vaudevilles, mis en scène en 1805 au théâtre du Vaudeville. Dans le jardin d'un cabaret de foire, Madame Baron (référence explicite à la directrice d'une des compagnies les plus importantes de l'Opéra-Comique) accueille Lesage, Fuzelier et D'Orneval. Nous sommes à la veille de la première d'Arlequin, roi de Serendib de Lesage (réellement représentée en 1713 à la foire de Saint-Germain), lorsqu'un nouvel interdit frappe les auteurs, qui commentent ainsi la censure :

Fuzelier : Ma foi, je sais gré à la Comédie-Française de nous avoir fait défendre de parler dans nos pièces, cela nous force à toujours chanter.

Le Sage : Et cela nous débarrasse de longues phrases ${ }^{14}$.

Bien que l'interdit soit décrété par un représentant de l'ordre public, la pièce est mise en scène à travers une échappatoire très originale : « D'abord on recevra le public, - explique Lesage - on lèvera la toile, les acteurs paraîtront sans rien dire. Les couplets seront reportés sur les écriteaux et lus par le public ; tandis que les acteurs seront muets $"{ }^{15}$. Avec cette pièce, le théâtre de boulevard ne se limite évidemment pas à célébrer ses origines, il thématise aussi, avec une conscience remarquable, la dramaturgie foraine à travers le dispositif du théâtre dans le théâtre et le recours à des citations de quelques passages tirés de la comédie originale de Lesage.

Le répertoire mixte du théâtre des Funambules peut être considéré comme l'expression principale de l'entrecroisement dramaturgique propre au théâtre populaire français des XVIII ${ }^{\mathrm{e}}$ et XIX ${ }^{\mathrm{e}}$ siècles. Inauguré en 1816 par Bertrand père et fils dans l'ancienne salle du Théâtre des Chiens Savants, il offre, bien avant d'accueillir la révolution du Pierrot-Deburau, des spectacles de la tradition foraine : acrobaties, pantomimes, parades, spectacles de marionnettes et exhibitions d'animaux dressés. Du large et multiforme répertoire de ce théâtre, qui sera démoli

Belin, Paris 2014 ; avec C. Triolaire (dir.), Enseigner la Révolution française. Héritages et actualité, Belin, Paris 2015, et Aux origines du théâtre patriotique, CNRS Éditions, Paris 2017.

14 P.Y. Barré, J.B. Radet et F.G. Desfontaines, Les Écriteaux, ou René Le Sage à la Foire de Saint-Germain : pièce anecdotique en deux actes et en prose, mêlée en vaudevilles, Léopold Collin, Paris 1806, p. 20 (acte I, scène 7).

15 Ibidem, p. 33 (acte II, scène 4). 
en 1862 à l'occasion des travaux de renouvellement urbanistique de Haussmann, demeure toutefois une petite partie $^{16}$.

La pantomime Ma mère l'oie ou Arlequin et l'œuf d'or (1830) de Lambert et Eugène $\left[* * *{ }^{*}\right]^{17}$ est certainement représentative de la dramaturgie des Funambules ${ }^{18}$. Il s'agit d'une féerie, qui évoque la comédie de Claude-Ignace de Barante et Charles Dufresny, Les Fées ou Contes de ma mère l'Oye (1697), mise en scène par les acteurs italiens un mois après la publication du recueil de Charles Perrault. La représentation suit le schéma traditionnel italien : Cassandre, Pierrot et un acteur comique cherchent à empêcher l'union entre Arlequin et Colombine. La course-poursuite des amoureux devient une spirale d'événements surréalistes, parfois obscurs, accompagnés des lazzi de la tradition italienne. Les personnages se pourchassent en traversant les paysages les plus variés : ils rivalisent dans une vaste plaine, se promènent le long d'une plage vénitienne, se cachent au milieu des arbres d'une forêt enchantée, pour trouver enfin le repos dans une taverne de province. Leur course ne finit jamais et reprend toujours depuis le début grâce à la batte d'Arlequin, qui résout chaque situation en faveur des amoureux. Les transformations spectaculaires opérées par Arlequin confirment le lien avec la tradition italienne. La baguette magique au service du personnage est, en effet, un thème récurrent des représentations de l'ancien

16 Sur les théâtres de boulevard, voir en particulier M. Albert, Les Théâtres des boulevards (1789-1848), Société Française d'Imprimerie et de Librairie, Paris 1902 ; idem, Les Théâtres de la Foire (1660-1789), Hachette, Paris 1900 ; H. Beaulieu, Les Théâtres du boulevard du Crime : cabinets galants, cabarets, théâtres, cirques, bateleurs : de Nicolet à Déjazet (1759-1862), Bibliothèque du vieux Paris, Paris 1905 ; B. Brunet, Le Théâtre de Boulevard, Armand Colin, Paris 2005 ; G. Cain, Anciens théâtres de Paris. Le Boulevard du Temple, les théâtres du boulevard, Charpentier Fasquelle, Paris 1906 ; A. Chaudet, Les Théâtres de Paris, Librairie Nouvelle, Paris 1859 ; M. Corvin, Le Théâtre de boulevard, Presses universitaires de France, Paris 1989 ; P. Gascar, Le Boulevard du crime, Hachette-Massin, Paris 1980 ; L.H. Lecomte, Histoire des théâtres de Paris, Daragon, Paris 1905-1912 ; J. McCormick, Popular Theatres of Nineteenth Century France, Routledge, London 1993 ; E. Mazzoleni, Migrazioni drammaturgiche. I teatri del Boulevard du Temple (1759-1862), Aracne, Roma 2017 ; C. Molinari, « Il boulevard parigino », [dans :] Storia del teatro, Laterza, Bari 1996, pp. 203-214, et J.C. Yon, S. Hartl et O.D. Mayer, Théâtres parisiens. Un patrimoine du XIXe siècle, Citadelles et Mazenod, Paris 2013. À propos du théâtre des Funambules, voir en particulier J. Janin, Deburau, histoire du théâtre à quatre sous, pour faire suite à l'histoire du Théâtre français, Librairie des bibliophiles, Paris 1832 ; A. Maurice, Les Théâtres des boulevards (1789-1848), Société Française d'Imprimerie et de librairie, Paris 1902 ; E. Mazzoleni, Pierrot sur scène. Anthologie de pièces et pantomimes françaises du XIX $X^{e}$ siècle, Classiques Garnier, Paris 2015 ; G. de Nerval, Le Boulevard du Temple. Spectacles populaires, coll. "Bibliothèque de la Pléiade ", Gallimard, Paris 1989 [L'Artiste 1, 3-12 mai 1844]; L. Péricaud, Le Théâtre des Funambules. Ses mimes, ses acteurs et ses pantomimes, depuis sa fondation jusqu'à sa démolition, Léon Sapin, Paris 1897. Le répertoire du mime J.B. Deburau a été édité par É. Goby (Pantomimes de Gaspard et Charles Deburau, Dentu, Paris 1859).

17 Nous signalons les noms des auteurs tels qu'ils figurent dans le manuscrit : Lambert et Eugène $[* * *]$. Ce dernier en particulier reste anonyme.

18 Pour l'édition moderne de la pantomime Ma mère l'oie ou Arlequin et l'œuf d'or (1830), publiée pour la première fois par Goby, voir notre Migrazioni drammaturgiche. 
Théâtre Italien exploité aussi par les auteurs français liés aux compagnies italiennes, tels Regnard et Dufresny. Dans Ma mère l'oie ou Arlequin et l'œuf d'or, la batte est un objet magique autour duquel tournent toutes les actions :

L'Aubergiste prépare une table pour Arlequin et Colombine, qui viennent y prendre place dès que le souper est servi. Ils sont bientôt dérangés par l'arrivée de Cassandre, du comique et de Pierrot. Les deux premiers s'empressent de prendre la place des amants. Arlequin les touche de sa batte : leurs jambes tombent, et ils ne peuvent plus bouger. Arlequin emmène Colombine, puis il fait la paix avec Cassandre et Pierrot. Alors il fait revenir leurs jambes. Ils en font usage pour s'élancer sur Arlequin, qui cherche à s'échapper. Les poursuivants lui barrent le passage. N'ayant plus d'autre ressource, il saute à travers la carte de géographie placée au-dessus de la cheminée. Elle se trouve aussitôt remplacées par un tableau sur lequel on lit: $\mathrm{ADIEU}^{19}$.

Dès lors, la scène change à nouveau et atteint son apogée en s'ouvrant sur un théâtre forain :

Une grande parade a lieu à la porte de la baraque des animaux vivants. Tout le monde y entre à l'exception d'Arlequin et de Colombine, qui jettent leurs déguisements. Sur un signe d'Arlequin, on voit sortir tous les spectateurs qui étaient entrés dans la baraque ; ils sont poursuivis par les animaux dont quelques-uns font des scènes comiques avec Cassandre et Pierrot ${ }^{20}$.

La course des personnages masqués, qui s'interrompt seulement avec l'arrivée d'une fée et la découverte d'un œuf d'or, est une métaphore du cercle formé par le récit, auquel le titre de la pantomime (Ma mère l'oie) fait allusion : une évocation aussi bien à la pièce de Barante et Dufresny qu'au recueil de Perrault, qui est un des schémas narratifs traditionnels. Mais elle représente aussi les migrations des théâtres forains, qui ont produit des spectacles mixtes capables d'influencer même des scènes futures et lointaines (voir certaines théories du $\mathrm{XX}^{\mathrm{e}}$ siècle et certains répertoires exportés outre-mer) grâce à l'entrecroisement de toutes les formes scéniques jusque-là séparées (danse, dialogue et musique). Dans cette optique, le théâtre populaire révèle une dramaturgie hautement expérimentale, qui porte un défi au théâtre officiel et en même temps contribue à sa réforme, tout en restant cachée. Denis Carolet, un auteur de la Foire très prolifique, reconnaissait déjà la valeur (artistique et morale) des tréteaux populaires. Dans le prologue de son Théâtre de la Foire (1734), il affirme : "L'intérêt qui l'a [le théâtre de la Foire] toujours rendu la victime des autres Théâtres, a souvent servi de voile à leur jalousie ; la difficulté de traiter ce genre de Comédie mixte, leur a fait craindre, avec raison, que d'habiles plumes s'en mêlant, ce Théâtre ne devînt, comme en effet il l'a été, le Théâtre des honnêtes gens $»^{21}$.

19 Lambert et Eugène, Ma mère l'oie ou Arlequin et l'œuf d'or, [dans :] E. Mazzoleni, Migrazioni drammaturgiche..., p. 55 (tableau VIII, scène 5).

${ }^{20}$ Ibidem, p. 57 (tableau X, scène 7).

21 D. Carolet, Le Théâtre de la Foire ou l'Opéra-Comique. Contenant une partie des pièces qui ont été représentées aux Foires de Saint-Germain et de Saint-Laurent pendant les années 1732, 1733 et 1734, vol. IX, Prault, Paris 1734, pp. 1-2. Dans la préface, l'auteur affirme que ses pièces suivent l'exemple de celles de Lesage, de D'Orneval et de Fuzelier. Ce recueil, accompagné d'airs 


\title{
THE TRICKERIES OF THE FOIRE: A SOCIOPOLITICAL AND THEATRICAL REVOLUTION
}

\author{
Summary
}

Between the $17^{\text {th }}$ and $19^{\text {th }}$ centuries, the competition between the official Parisian theatres and the popular trestles, such as the lodges of the fairs and the stages of the Boulevard, contributed to the development of an experimental dramaturgy characterised by theatrical crossing and the primacy of the actor's gesture. To circumvent the prohibitions imposed by the Comédie-Française and the Opera, the impresarios of the Fair elaborate dramaturgical strategies full of invention, based on shows with simple units suitable for the combination of different genres. This theatre is thus able to develop into a stable artistic system, while remaining in a context of marginality and uncertainty; and to inspire some twentieth-century theories on the actor's body, such as those of Edward Gordon Craig and Jacques Copeau. Focused on documentary archive evidence, this paper aims to bring out the main aspects of the unofficial repertoires, which turn out to be places of socio-political confrontation and, at the same time, systems of propagation of aesthetic reforms.

Key words: $18^{\text {th }}$ - and $19^{\text {th }}$-century French theatre, theatre of the Foire, Commedia dell'Arte, theatrical crossings, censorship, artistic innovation.

et d'illustrations signées par Carolet même, est très riche. Il contient Le Reveil de l'Opéra-Comique (Foire de Saint Laurent, 1732) qui, comme beaucoup d'autres pièces, met en scène la personnification de la Foire occupée à attirer le public après une longue période de fermeture imposée par le Parlement, et L'Amour désœuvré ou les vacances de Cythère attestant le lien entre le théâtre forain et celui des Italiens. Nous voici à la Foire, où un acteur italien s'entretient avec Vénus. Il lui avoue, en s'exprimant seulement à travers des couplets chantés, ses inquiétudes à l'égard de son théâtre (le public est trop rare et corrompu). 